\title{
ECOLOGÍA Y CONSERVACIÓN DE CENTAURIUM SOMEDANUM M. LAÍNZ (GENTIANACEAE), PLANTA ENDÉMICA DE LA CORDILLERA CANTÁBRICA (ESPAÑA) $^{1}$
}

\author{
B. JIMÉNEZ-ALFARO G., A. BUENO SANCHEZ \& J. A. FERNÁNDEZ PRIETO
}

Jardin Botánico Atlántico. Universidad de Oviedo Aoda. del Botánico, s/n. E-33394 Gijón (Asturias) jimerezalfarogutionies

\begin{abstract}
Centaurium somedanum is a cantabrian endemism, with less than twenty localities known in the western Cantabrian Mountains. In order to assess its conseroation status, a short-term study has been developed, by applying a Basic Study and an Habitat Integrated Study, which have defined the habitat for the plant, number of populations and some demographic information. The results from the study have confirmed the extinction of the plant in some localities where it had been recorded. $\mathrm{C}$. somedanum is a short-lioed chamaephyte plant, limited to fourteen populations at present, and only four of them exced froe hundred aduit individuals. They occur on very specific habitats, in the surroundings of calcareous hard water springs, between 500 and 1600 melers of altitude. The specific nature and scarcity of its habitat are the main reasons of the rareness and threat of this species. Some other rare species and plant communities from Cantabrian Mountains share with $C$. somedanum the need of this type of spring communities conservation, which are currently under different threats.
\end{abstract}

Key-words: Centaurium somedanum, Cantabrian Mountains, Plant Conservation, Habitat, Spring communities.

RESUMÉ-Centaurium somedanum est un endémisme cantabrique présent dans moins d'une vingtaine de localités situées da l'extrêne ouest des Monts Cantabres. Pour mienx connaitre son êtat de conservation à court terme, on a developpé une Étude Basique et une Étude Integrée de l'habitat, en définissant la disponibilité de l'environnement adéquat pout la plante et son rapport azec son état

1 Recibido: 4-VIII-2005. Aceptaso: 19-X-2005. 
PIRINEOS 160

\begin{abstract}
de conservation. Les résultats de l'étude nous ont permis de constater la tisparition de l'espèce dans quelques localités où eile avait été reportée. C. somedanum est une chaméphyte de oje courte, actuellement rétuite à quatorze populations, dont seulentent quatre arrivent à dépasser cings cents individus. Elles occupent des habitats très specifiques aux alentouts de sources d'eaux carbonalées, entre 500 et 1600 mètres d'altitude. La nature spécifique de son habitat et le manque de celui-ci semblent le principales causes de la rareté et la fragilité de lespèce. Avec $\mathrm{C}$. somedanum, d'autres espèces et communautés végétales rares des Monts Cantabres nous signalent la nécessité de conserver les milievix lies à ce type de sources, maintenant soumises à différentes menaces.
\end{abstract}

Mots clé: Centaurium somedanum, Monts Cantabres, Conservation végétale, Habitat, Communautés fontinales.

\footnotetext{
RESUMEN- Centaurium somedanum es un endenismo cantabrico conocito en menos de veinte localidades del extremo occidental de la Cordillera Cantábrica. Con el fin de conocer su estado actual de conservación, se han realizado un Estudio Básico y un Estudio integrado del Hábitat, definiendo los requerimientos del hábitat de la planta, el múmero de poblaciones y su estado dentográfico. Los resultados del estudio han permitido constatar la desaparición de la especie en algunas de las localidades en que habia sido citada. C. somedanum es un caméfito de vida corta, del que actualmente se conocen catorce poblaciones, y de eilas sólo cuatro superan los quinientos inditiduos. Ocupa habitats moty especficos, en torno a surgencias de aguas carbonatadas, desde 500 a $1600 \mathrm{~m}$ de atitud. La especificidad y escasez de su hábitat son las principales causas de su rareza y ameraza. Junto a $\mathrm{C}$. somedanum, otras especies y comunidades pegetales raras de la Cordillera Cantábrica sentalan la necesidad de conseruar los ambientes ligados a este tipo de surgencias, actualmente sometidas a diferentes amenazas.
}

Palabras clave: Centaurium somedanum, Montes Cantábricos, Conservación vegetal, Hăbitat, Comunidades fontinales.

\title{
1. Introducción
}

La flora endémica de la Cordillera Cantábrica está representada por al menos 50 taxones (DÍAZ GONZÁLEZ \& FERNÁNDEZ PRIETO, 1994), cuyo origen evidencia una fuerte influencia paleo-mediterránea y alpino-pirenaica (RIVAS-MARTÍNEZ et al, 1984), La historia botánica y el clima de esta región montañosa eurosiberiana, en transición hacia la región mediterránea, favorece la existencia de plantas endémicas de orígenes filogenéticamente muy diversos. El género Centaurium Hill (Gentianaceae) presenta en la Península Ibérica un importante centro de endemicidad, con relación a su principal área de distribución, la región mediterránea (ZELTNER, 1970; MANSION \& STRUWE, 2004). Su concepción sistemática actual y su área básicamente pale- 
ártica y fundamentalmente mediterránea (MANSION, 2004) acentúan aún más la relevancia de la Península lbérica como principal centro de diversidad y especiación del género.

Centaurium somedanum M. Laínz, Bol. Ins. Est. Ast., ser. C., 22: 32 (1976), es el único representante del género cuya área de distribución se limita a una región montañosa de clima templado. Se trata de un endemismo cantábrico exclusivo de la parte occidental de la Cordillera Cantábrica (subprovincia Orocantábrica, sector Ubiñense-Picoeuropeano, subsector Ubiñense), territorio situado entre las provincias de León y Asturias, donde dominan los sustratos calcáreos y se concentra el mayor número de plantas endémicas de esta cordillera (RIVAS-MARTINEZ et al, 1984). C. somedanum muestra afinidad morfológica con C. chloodes, por lo que ROMERO (1977) la propuso como $C$. chloodes (Brot.) subsp. somedanum (M. Laínz) Romero. Su área de distribución es inferior a $500 \mathrm{~km}^{2}$, con menos de veinte localidades conocidas.

Respecto a su grado de amenaza, se considera esta especie en la categoria de Vulnerable, VU:D2, según los criterios de UICN de 1994 (VV. AA., 2000). No ha sido incluida, para su actualización, en el Atlas y Libro Rojo de la flora española (BAN̄ARES et al., 2003), por lo que la clasificaçión actual se basa únicamente en trabajos de recopilación bibliográfica. Debido a su rareza, es tratada como "Taxón prioritario" en la Directiva Hábitats (Anexo II, Directiva 97/62/CEE), $y$ "Sensible a la alteración del hábitat" en el Catálogo Regional de Especies Amenazadas del Principado de Asturias (Decreto 65/95 de esta Comunidad Autónoma).

Si bien se trata de uno de los endemismos cantábricos más escasos y de área más restringida, no existen estudios sobre su biología, ecología o corología precisa, información que resultaría esencial para establecer el grado de amenaza en función de los criterios UICN (2001), y debería condicionar futuras medidas de conservación y gestión. Por ello, el presente estudio tiene como objetivo analizar los aspectos biológicos y ecológicos básicos de $C$. somedanum que permitan establecer su estado de conservación y definir las principales amenazas a que está sometido.

\section{Material y método}

\subsection{Caracteristicas generales de la planta}

Centaurium somedanum es una planta perenne de $3-10 \mathrm{~cm}$ de altura, monoica, con flores hermafroditas y corola rosado-púrpura. La floración tiene lugar en el mes de julio ( \pm 20 días, en función de la altitud). El número de flores es de 
(1) 2-4 (15), y forman un corimbo. La autogamia es frecuente en las especies del género Centaurium, si bien en muchos casos se acompaña de polinización cruzada por medio de insectos. La elevada proporción de frutos por individuo y la frecuente visita de esos invertebrados hacen pensar que en C. somedanum también se dan tales procesos. El fruto es una cápsula septicida que madura alrededor de un mes después de la floración.

C. somedanum vive en torno a manantiales y paredes rezumantes, entre 500 y 1600 metros de altitud. Los conocimientos fitosociológicos existentes indican una especial afinidad por las comunidades de fuentes carbonatadas:

surgencias de las alianzas Cratoneurion commutati Koch 1928 y Adiantion capilli-veneris Br.-Bl. ex Horvatic 1934 (DÍAZ GONZÁLEZ et al,, 2003).

turberas planas basófilas del orden Caricetalia davallianae Br-Bl. 1949 (ROMERO, 1977).

-herbazales húmedos, travertinos o bordes de arroyos, es decir, comunidades de transición entre las clases Adiantetea $\mathrm{Br}$. Bl. y Montio-Cardamintetea $\mathrm{Br}$. Bl. \& Tüxen (FERNÁNDEZ PRIETO, 1978).

Las amenazas descritas para la planta se refieren a la alteración del aporte de agua a sus hábitats y a la proximidad de carreteras a sus poblaciones (DÍAZ GONZÁLEZ et al., 2003).

\subsection{Visita y estudio de las poblaciones}

El estudio comenzó con la revisión de la corología de la planta a partir de datos bibliográficos, trabajos inéditos y comunicaciones personales. Todas las citas recogidas fueron incorporadas a una base de datos, y ligadas a un Sistema de Información Geográfica (SIG), lo que permitió obtener un primer mapa de la distribución de $C$. somedanum -un total de 17 localidades-el cual se utilizó para planificar los estudios de campo. Las poblaciones de C. somedanum encontradas fueron estudiadas siguiendo la metodología de "aproximación rápida" (MARRERO al. in BANAARES, 2002; MARRERO al, 2003), propuesta para realizar estudios de conservación en plazos cortos, no superiores a dos años; esta técnica permite obtener datos suficientes para la evaluación preliminar del estado de conservación de una especie, asignándole, llegado el caso, una determinada categoría de amenaza. También se ha aplicado la metodología propuesta en el estudio de la flora amenazada de España (BAÑARES et al., 2003), cuyo Estudio Básico plantea recoger datos sobre distribución, demografía, biología y comportamiento ecológico del taxón en estudio. Se han incorporado, además, datos relativos a la ecología mediante un Estudio Integrado del Hábitat, siguiendo las líneas metodológicas iniciadas por MENGES \& GORDON (1996) o KEITH (1998), aplicadas después por

48 
VALADON (2003), donde el conocimiento del hábitat se considera fundamental para el análisis inicial del estado de conservación de plantas.

\subsection{Estudio Básico}

Corologia.- Las 17 localidades visitadas fueron muestreadas en función del grado de precisión de las citas, localizándolas mediante GPS (Global Positional System) y verificando la presencia o ausencia de la planta. Los datos fueron volcados a un SIG (ArcView 3.2), obteniendo un mapa de distribución actual confirmada, y calculando la extensión de presencia, según la definición UICN (2001), mediante el método del Mínimo Polígono Convexo (MPC), y el de la media armónica (DIXON \& CHAPMAN, 1980), cálculo espacial no paramétrico que permite establecer áreas de inter-proximidad entre poblaciones. También se determinó la "extensión de presencia" (mediante el MPC) y el "área de ocupación" (midiendo la superficie real ocupada) en cada una de las poblaciones, definidas en correspondencia con el concepto biológico de población.

Demografía- Los censos realizados fueron agrupados en función de las poblaciones definidas, a partir del análisis de las distancias interpoblacionales y las características de la planta. Cuando la ubicación de la población no permitía un muestreo completo, se realizaron censos por estimación a partir de parcelas representativas en las que se determinó la densidad media, aphicándola después al área de ocupación de cada población. En todos los casos se contaron individuos visibles con una talla mínima de $2 \mathrm{~cm}$-juveniles y adultos-.

Biología y ciclo de vida.- Además de la obtención de datos sobre la fenología y reproducción de la planta, se marcaron todos los individuos de una subpoblación representativa correspondiente a una parcela de $20 \mathrm{~m}^{2}$ en la localidad de La Malva; estos individuos fueron seguidos durante los años 2003 y 2004 , con el fin de definir el ciclo de vida de la planta. Los individuos $(\mathrm{N}$ inicial = 27; $\mathrm{N}$ final $=30$ ) fueron fotorreferenciados con una marca distintiva, tomando datos sobre su tamaño y estado de desarrollo, segín los siguientes estados: Plántula (P); No reproductor (N), que incluye estado juvenil (aparición de ramificaciones caulinares) o adulto; y Adulto reproductor $(\mathrm{R})$.

\subsection{Estudio Integrado del Hábitat}

Se basó en la autoecología de la planta y en la sinecología de las comunidades donde se halla. 
Autoecologia- A lo largo del área de ocupación de las poblaciones se tomaron datos topográficos (altitud, exposición y pendiente) y edáficos (profundidad y naturaleza del suelo, humedad y $\mathrm{pH}$ ).

Caracterización fitosociológica.- Se tomaron inventarios fitosociológicos en áreas de superficie reducida (entre 1 y $10 \mathrm{~m}^{2}$ ), seleccionando micro-comunidades de $C$. somedanum, y muestreándolas según el criterio de homogeneidad (BRAUN-BLANQUET, 1979). También se revisaron inventarios publicados de las comunidades más afines a las inventariadas, seleccionadas en función de la presencia de especies características. Todos los datos fueron procesados mediante análisis multivariante de tipo DCA (Detrended Correspondence Analysis) a través del programa CANOCO (LEPS \& SMILAUER, 2003). Posteriormente fureron clasificados mediante el método TWINSPAN - Twin Indicator Species Analysis, cf. HILL (1979)-, elaborando tablas sintéticas con valores de fidelidad y frecuencia de especies, mediante el programa JUICE 6.2 (TICHY, 2002).

\section{Resultados}

\subsection{Estudio Básico}

\section{Corologia}

El área de distribución actual de $C$. somedanum se reduce a las provincias de Asturias (municipio de Somiedo) y León (municipio de Cabrilanes). No se encontró la planta en 6 de las 17 localidades donde se había señalado su presencia, coincidentes con el extremo nororiental de su distribución (Figura 1). Además, en el presente estudio se descubrieron 7 de esas localidades (Tabla 1).

En algunas localidades donde no se encontró la planta se constató la desaparición de los ambientes referidos en la bibliografía (fuentes o manantiales), debido a la construcción de infraestructuras públicas de comunicación. Este fue el caso de: Villanueva (Teverga), citada por LAINZ (1976), AEDO (1990) y FERNÁNDEZ CASAS et al. (1994), más Sograndio (Proaza) y Sellón de San Martín (Belmonte), citadas por LASTRA (1989). En otros casos faltaba precisión o había errores en las coordenadas UTM, pero las localidades fueron igualmente prospectadas, aunque sin éxito. Tal es el caso de la ya citada Villanueva (Teverga), AEDO (1990), el Barranco de Cavadura (Grado), citado por LASTRA (1989) y La Riera (Somiedo), referida por LAÍNZ (l. c.), que podría corresponder a la población de La Malva. 


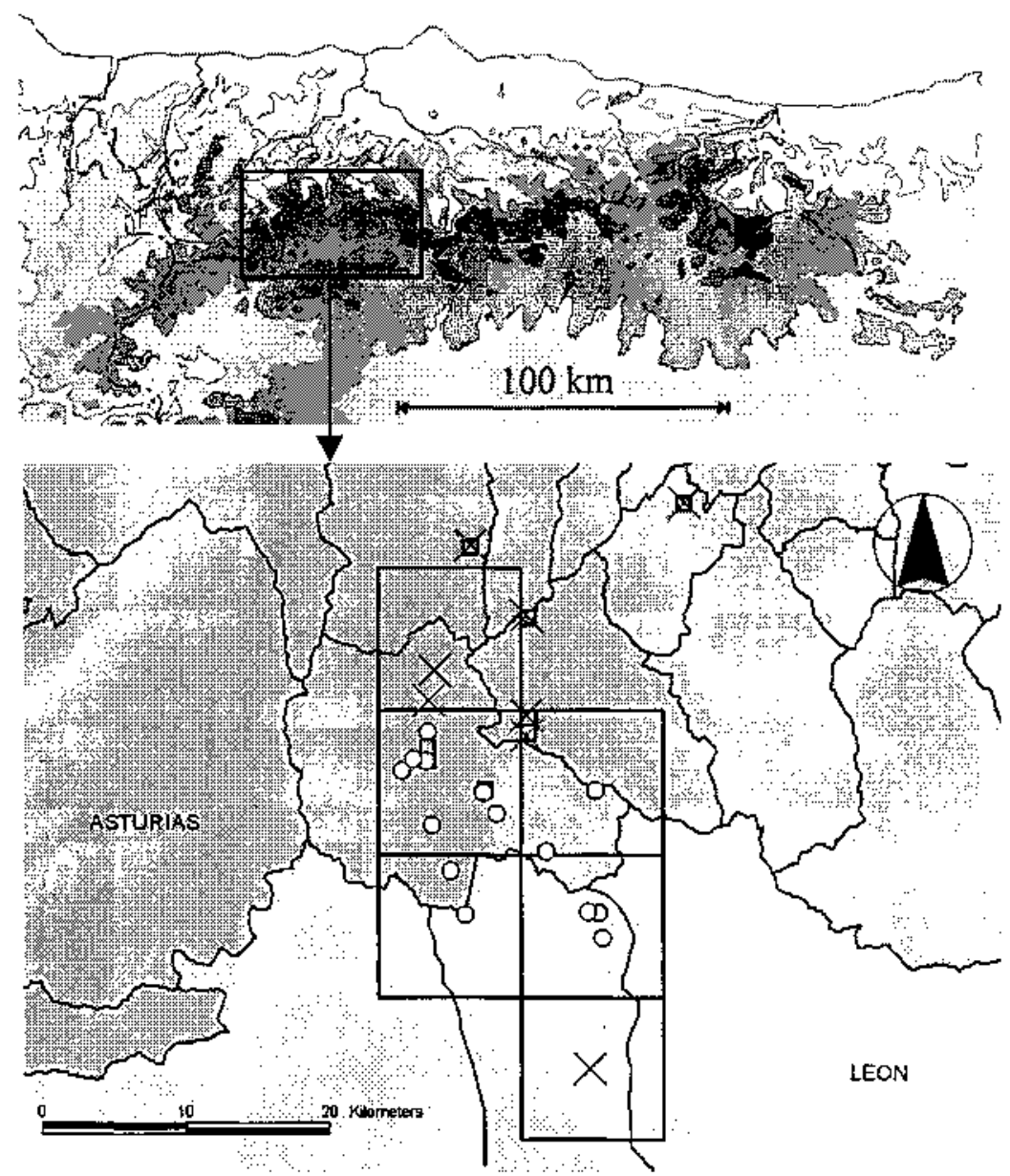

Figura 1. Mapa de distribución actual confirmada de C. somedanum y cuadrículas UTM de $10 \times 10 \mathrm{~km}$ y $1 \times 1 \mathrm{~km}$ de las localidades y citas revisadas (cuadrados). Se indica la presencia (o) o ausencia $(X)$ de la planta en las zonas visitadas.

Figure 1. Present distribution map of C. somedanum and $10 / 1 \mathrm{~km}$ UTM grids from visited localities. Plant occurrence ( $O$ ) or absence $(X)$ are indicated in visited localities.

El Mínimo Polígono Convexo que engloba las localidades donde C. somedanum está presente, permite definir una "extensión de presencia" de $134 \mathrm{~km}^{2}$ (Figura 2). El análisis espacial resultante del método de la media armónica, de tipo cluster, permite distinguir áreas de mayor proximidad interpoblacional, 
en función de los valores de distancias mínimas seleccionados. A partir de la rasterización de celdas para 150 y 75 metros de lado, los mapas resultantes (Figura 2) ofrecen resultados análogos, indicando una mayor conexión de las poblaciones noroccidentales, que corresponden a la vertiente norte de la Cordillera Cantábrica, cuyo eje central no parece representar un aislamiento relevante para la especie. Asumiendo una distancia mínima de $0,5 \mathrm{~km}$ para diferenciar unidades poblacionales, en función de las características y distribución de la especie, se pueden establecer 14 poblaciones (Figura 2), considerando una de ellas como una metapoblación ( $\mathrm{N}^{\circ} 1$ - La Malva), de $2 \mathrm{~km}^{2}$ de extensión. Las distancias absolutas entre las poblaciones así definidas varian entre 0,7 y $20 \mathrm{~km}$, con una media de $2,2 \mathrm{~km}$. En tres situaciones las distancias interpoblacionales están próximas al kilómetro (distancias de 0,$7 ; 0,9$ y 1,1 $\mathrm{km}$ ), por lo que su aislamiento genético no puede ser determinado, en ausencia de datos precisos sobre la reproducción, dispersión o variabilidad genética de la especie. Si bien las poblaciones definidas se consideran las más apropiadas para su estudio y caracterización, algunas de ellas presentan mayor posibilidad de compartir procesos de migración, en función de su proximidad geográfica (Figura 2).
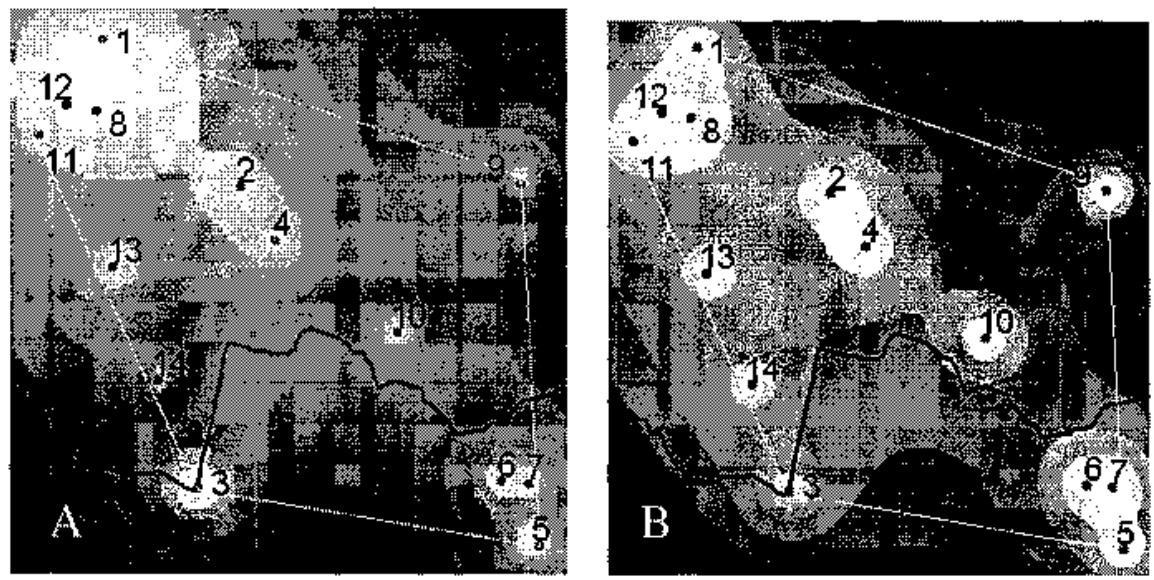

Figura 2. Extensión de presencia de las 14 poblaciones estudiadas, mediante el MPC (polígono, $134 \mathrm{Km}^{2}$ ) y mediante el método de la media armónica (mapa isométrico), a partir de celdas de $150 \mathrm{~m}$ (izquierda) y $75 \mathrm{~m}$ (derecha) de lado. La línea central corresponde al límite entre las provincias de Asturias y León.

Figure 2. Extent of occurrence from the I4 studied population, using MPC method (polygon, $134 \mathrm{~km}^{2}$ ) and Harmonic Mean method (isometric map), in order to grids of $150 \mathrm{~m}(A)$ and $75 \mathrm{~m}$ (B). It shows dividing line of Asturias and León provinces. 


\section{Demografia}

El número de individuos de cada población no guarda relación aparente con el "área de ocupación" o la "extensión de presencia" poblacional (Tabla 1), debido a su irregular distribución. El hábitat en que viven se desarrolla

Tabla 1. Datos demográficos y geográficos de las poblaciones visitadas.

Table 1. Demographic and geographic data for visited populations.

\begin{tabular}{|c|c|c|c|c|c|c|c|}
\hline $\mathbf{P}$ & LOC & REF & UTM & $\overline{A L T}$ & $\mathbf{N}$ & $\mathbf{E P}$ & $\mathrm{AO}$ \\
\hline 1 & $\begin{array}{c}\text { La Malva, } \\
\text { Somiedo (AS) }\end{array}$ & $3,5,6,9$ & $\begin{array}{c}29 \mathrm{TQH} \\
2376 \\
29 \mathrm{TQH} \\
2377 \\
29 \mathrm{TQH} \\
2378\end{array}$ & 700 & $\begin{array}{l}577 \\
\text { (D) }\end{array}$ & 213.000 & 792 \\
\hline 2 & Bobias, Somiedo (AS) & 9 & $\begin{array}{c}29 \mathrm{TQH} \\
2691\end{array}$ & 1.100 & $\begin{array}{l}173 \\
\text { (D) }\end{array}$ & 400 & 170 \\
\hline 3 & $\begin{array}{l}\text { (") El Puerto, } \\
\text { Cabrillanes (LE) }\end{array}$ & - & $\begin{array}{c}29 \mathrm{TQH} \\
2665\end{array}$ & 400 & $\begin{array}{l}380 \\
\text { (D) }\end{array}$ & 2.120 & 500 \\
\hline 4 & $\begin{array}{l}\text { (*) Valle del Lago, } \\
\text { Somiedo (AS) }\end{array}$ & 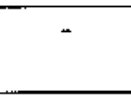 & $\begin{array}{c}29 \mathrm{TQH} \\
2872\end{array}$ & 1.260 & $\begin{array}{l}425 \\
\text { (D) }\end{array}$ & 434 & 300 \\
\hline 5 & $\begin{array}{c}\text { Torre 1, } \\
\text { Cabrillanes (LE) }\end{array}$ & $\begin{array}{c}1,2,4 \\
7,8\end{array}$ & $\begin{array}{c}29 \mathrm{TQH} \\
3466\end{array}$ & 1.450 & $\begin{array}{l}303 \\
\text { (D) }\end{array}$ & 6.300 & 400 \\
\hline 6 & $\begin{array}{c}\text { Torre } 2, \\
\text { Cabrillantes (LE) }\end{array}$ & $\begin{array}{c}1,2,4 \\
7,8 \\
\end{array}$ & $\begin{array}{c}29 \mathrm{TQH} \\
3466 \\
\end{array}$ & 1.600 & $\begin{array}{l}155 \\
(\mathrm{D})\end{array}$ & 4.181 & 115 \\
\hline 7 & $\begin{array}{c}\text { Torre } 3, \\
\text { Cabrillanes (LE) }\end{array}$ & $\begin{array}{c}1,2,4 \\
7,8 \\
\end{array}$ & $\begin{array}{c}29 \mathrm{TQH} \\
3466 \\
\end{array}$ & 1.700 & $\begin{array}{l}220 \\
\text { (D) } \\
\end{array}$ & 631 & 85 \\
\hline 8 & $\begin{array}{l}\text { Monte Gurugú, } \\
\text { Somiedo (AS) }\end{array}$ & 9 & $\begin{array}{c}29 \mathrm{TQH} \\
2376\end{array}$ & 735 & $\begin{array}{c}16 \\
\text { (D) } \\
\end{array}$ & 3.800 & 50 \\
\hline 9 & $\begin{array}{l}\text { (*) Valle Arroxos, } \\
\text { Somiedo (AS) }\end{array}$ & 11 & $\begin{array}{c}29 \mathrm{TQH} \\
3574\end{array}$ & 1.250 & $\begin{array}{c}125 \\
0 \\
(\mathrm{E})\end{array}$ & 6.050 & 300 \\
\hline 10 & $\begin{array}{l}\text { (*) Murias Longas } \\
\text { Somtedo (AS) }\end{array}$ & 11 & $\begin{array}{c}29 \mathrm{TQH} \\
3169\end{array}$ & 1.550 & $\begin{array}{c}140 \\
0 \\
(\mathrm{E})\end{array}$ & 1.785 & 1.000 \\
\hline 11 & $\begin{array}{c}\text { (") Furán } \\
\text { Somiedo (AS) }\end{array}$ & 11 & $\begin{array}{c}29 \mathrm{TOH} \\
2175\end{array}$ & 830 & $\begin{array}{l}500 \\
\text { (E) }\end{array}$ & 50 & 50 \\
\hline 12 & $\begin{array}{c}\text { (*) Aquinu } \\
\text { Somiedo (AS) }\end{array}$ & I1 & $\begin{array}{c}29 \mathrm{TQH} \\
2276\end{array}$ & 880 & $\begin{array}{l}80 \\
\text { (D) }\end{array}$ & 20 & 20 \\
\hline
\end{tabular}


PIRINEOS 160

\begin{tabular}{|cccccccc|}
\hline P & LOC & REF & UTM & ALT & N & EP & AO \\
\hline & Caunedo, & & $29 T Q H$ & & $\mathbf{3 0 0}$ & & \\
13 & Somiedo (AS) & 10 & 2372 & 850 & $(\mathrm{E})$ & 670 & 600 \\
\hline & (*) Puerto 2, & & $29 T Q H$ & & 50 & & \\
14 & Somiedo (AS) & 11 & 2568 & 1.400 & (D) & 50 & 50 \\
\hline & TOTAL & & & & 6.849 & $\mathbf{2 3 9 . 4 3 1}$ & $\mathbf{4 . 4 3 2}$ \\
\hline
\end{tabular}

P: Población (Population); LOC: Localidades (Localities), (*): nuevas localidades (new localities); REF: Referencias (references); UTM: cuadriculas UTM (UTM squares), ALT: Altifud (altitude); N: Número de individuos (number of individuls), (D): censo directo (dinet censws), (E): estimado (estimaldd); EP: ( $\left.\mathrm{m}^{2}\right)$ - Exlensión de presencia (extent of occurrence); $\mathrm{AO}$; $\left(\mathrm{m}^{2}\right)$ - Area de ocupación (Aret of accupancy).

sobre biotopos poco uniformes, tratándose de un mosaico de diferentes micro-hábitats. Esto provoca una distribución contagiosa de los individuos, en función de las variaciones de humedad y sustrato, por lo que los censos por estimación deben ser tomados como meras aproximaciones. Además, se ha constatado una importante variación interanual en el hábitat disponible de algunas poblaciones, ya que en el momento de ser censadas mostraban una distribución diferente a la del año anterior, debido a las alteraciones del régimen de precipitaciones de cada año.

\section{Biologia y ciclo de vida}

El seguimiento de los individuos de la parcela de La Malva permitió confirmar el carácter perenue de la planta. Los individuos forman una roseta de 2-4 (6) $\mathrm{cm}$ de diámetro, ramificada cada año mediante extensiones laterales. Se trata de un caméfito de ciclo corto, con una vida media estimada de 3-6 años y con una alta mortalidad, inducida por las características del medio. El $75 \%$ de los individuos muertos correspondieron a ejemplares adultos reproductores (de 3-5 años de vida). El resto fueron plántulas de un año que no prosperaron. Los individuos nuevos, procedentes de semillas germinadas, son todos vegetativos, el primer año en forma de roseta (80\%) o con alguna ramificación (20\%). El $80 \%$ de las plántulas identificadas el primer año dieron lugar a rosetas ramificadas no reproductoras. La transición de un solo año no permite definir parámetros demográficos o matrices de transición, si bien ha servido para definir una propuesta del cíclo biológico de la planta a partir de los datos disponibles (Figura 3 ). 


\begin{tabular}{|lcccc|}
\hline & $\mathbf{S}(\mathbf{N i}=?)$ & $\mathbf{P}(\mathrm{Ni}=13)$ & $\mathbf{N}(\mathrm{Ni}=2)$ & $\mathbf{R}(\mathbf{N i}=12)$ \\
\hline $\mathbf{S}$ & $\vdots ?$ & 0 & 0 & 0 \\
$\mathbf{P}$ & 10 & 3 & 0 & 0 \\
$\mathbf{N}$ & 1 & 4 & 0 & 2 \\
$\mathbf{R}$ & 0 & 4 & 2 & 4 \\
\hline
\end{tabular}

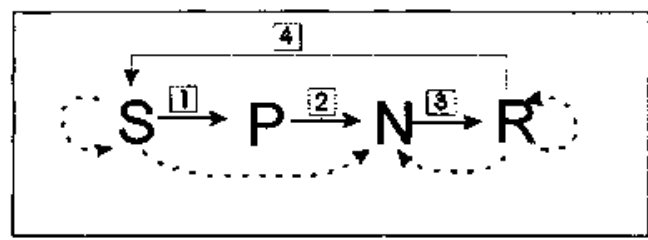

Figura 3. Transición observada durante un año (2003-2004) y ciclo de vida propuesto. $\mathrm{Ni}=27 ; \mathrm{Nf}=30$. (S: Semilla; P: Plántula; $\mathrm{N}$ : Juvenil o Adulto no reproductor; R: aduilto reproductor).

Figure 3. Transition data from one year (2003-2004) and vital cycle proposed. $\mathrm{Ni}=27$; $N f=30$. (S: Seed; P: Plantule; N: Non reproductor; R: reproductor).

Las semillas presentan un color pardo oscuro en la madurez, son lisas y no superan los $0,02 \mathrm{~mm}$ de diámetro. El número medio de semillas por cápsula es de 105,29 ( $\pm 47,98)$, según los datos obtenidos a partir de 27 frutos recolectados en cuatro poblaciones (números $1 ; 3 ; 4$ y 12 en la Tabla 1). No parecen existir mecanismos específicos de dispersión de semillas, salvo la gravedad o su arrastre por el agua, favorecido por su pequeño tamaño y peso. Se ha observado reproducción vegetativa por acodos en las ramas basales de algunos individuos, especialmente en ambientes rocosos.

\subsection{Estudio Integrado del hábitat}

\section{Autoecología}

Las poblaciones de C. somedanum estudiadas se desarrollan en el bioclima templado oceánico, en el piso supratemplado de tipo submediterráneo (RIVAS-MARTÍNEZ al., 2002), caracterizado por presentar una precipitación estival ligeramente reducida, coherente con su proximidad a territorios de clima mediterráneo. No existe una especial afinidad por determinada altitud - con un rango amplio, entre 500 y 1600 metros -, o exposición - muy varia- 
ble en las poblaciones visitadas: E- $48 \%$; ESE- $12 \%$; SSE- $10 \%$; SE- $8 \%$; S- 2 $\%$; SO- $6 \%$; en llano- $13 \%$ - En todos los casos las poblaciones se sitúan en ambientes despejados, sin cobertura arbustiva o arbórea, debido a las limitaciones del suelo (toboso en su mayor parte), y a la pendiente, en algunas poblaciones próxima al $100 \%$.

La afinidad ecológica de C. somedanum en las poblaciones visitadas se ajusta a factores edáficos, localizándose siempre alrededor de surgencias o fuentes de aguas frías y carbonatadas, donde es común la formación de toba. Son, por lo tanto, sustratos muy húmedos, con un cierto grado de encharcamiento y calcáreos, aunque a veces se dan en zonas de contacto litológico entre terrenos calizos y pizarrosos. Los valores de profundidad de sustrato medidos varían entre 1 y $15 \mathrm{~cm}$, con un promedio de $6,24 \mathrm{~cm}( \pm 3,38 ; \mathrm{N}=41)$. En la mayor parte de los casos $(86,6 \% ; N=67)$ estos sustratos se corresponden con complejos de carbonato cálcico precipitado y musgos embebidos en él, en diferentes fases desde la formación de toba a su petrificación como roca travertínica. Como corresponde a las altas concentraciones de carbonatos de estos ambientes, las medidas de $\mathrm{pH}$ son elevadas, entre 7,77 y 7,97 .

Algunos de los ambientes que rodean las surgencias se han identificado por la presencia constante de precipitados de carbonato cálcico, producidos por el repentino cambio físico del agua subterránea en su salida al exterior. Opuestamente, durante los trabajos de campo se localizaron fuentes próximas a las comunidades estudiadas, donde la ausencia de $C$. somedanum y otras especies parecía ir ligada a la ausencia del precipitado de carbonato cálcico. Las medidas de $\mathrm{pH}$ tomadas en las poblaciones de Torre de Babia (n. ${ }^{\circ}$, 6 y 7 de la Tabla 1) y en manantiales próximos fueron comparadas. Las diferencias (Figura 4) permiten establecer una relación entre la presencia de $C$. somedanum, las concentraciones de carbonatos y los valores de $\mathrm{pH}$ de dichos manantiales, a partir de las medidas tomadas en muestras de suelo y agua.

\section{Caracterización fitosociológica}

Para el análisis de 57 inventarios realizados en las poblaciones de $C$. somedanum se han incorporado 15 inventarios de origen bibliográfico, relacionados con las dos comunidades propias de surgencias descritas en la Cordillera Cantábrica: asociación Hyperico nummularii-Pinguiculetum coenocantabricae $\mathrm{T}$. E. Díaz, Guerra \& Nieto 1982 (orden Adiantetalia capilit-veneris Br.-BI. ex Horvatic 1934) y asociación Pinguiculo grandiflorae-Caricetum lepidocarpae Rivas-Martínez, T.E. Díaz, F. Prieto, Loidi \& Penas 1984 (orden Caricetalia davallianae). Entre estos inventarios se incluye el tipo de dichas comunidades 


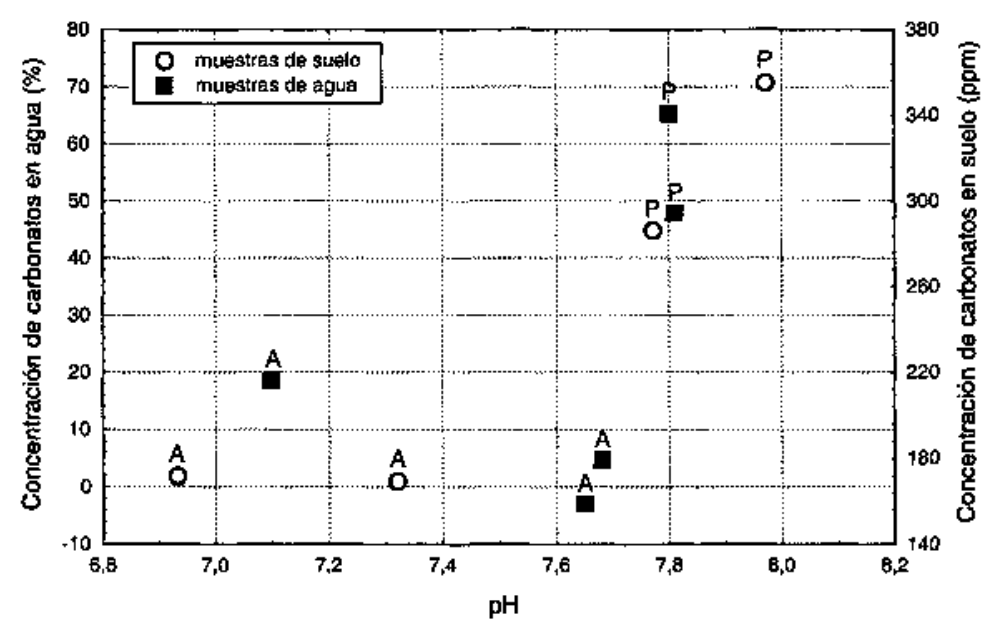

Figura 4. Concentración de carbonatos y medidas de $\mathrm{pH}$ en surgencias con presencia (P) y ausencia (A) de C. somedanum.

Figure 4. Carbonates and $\mathrm{pH}$ measure concentrations in springs with occurrence $(P)$, and in absence $(A)$ of $C$. somedantum.

y el de la subasociación Pinguiculo grandiflorae-Caricetum lepidocarpae equisetosum variegati, descrita precisamente en el área de estudio.

Los resultados del análisis multivariante (DCA) se muestran mediante un diagrama de ordenación (Figura 5). El valor más alto de longitud de gradiente, correspondiente al eje $x$, fue de 5,6, lo que sugiere la idoneidad del análisis unimodal (LEPS \& SMILAUER, 2003). Dicha figura permite apreciar un gradiente, desde los grupos más afines a los inventarios del orden Adiantetalia capilli-verneris - situados en el extremo izquierdo del eje $x-$, hacia aquellos más próximos al orden Caricetalia davallianae - a la derecha del mismo eje -, los cuales definen los dos extremos de la variación total.

La correlación con las variables ambientales de altitud y pendiente (eje $x$ ) resultó altamente positiva $(\mathrm{r}=0.961)$, lo que permite explicar la frecuencia de aparición de algunas especies, si bien ello no implica que sean éstos los únicos factores determinantes. Por otro lado, los datos referentes a la exposición (eje y), no presentan valores altos de correlación $(\mathrm{I}=0,434)$.

Entre las especies indicadoras de las distancias estadísticas entre muestras (Figura 6), cobran especial relevancia los briófitos, como Eucladium verticillatum, Pellia sp. pl. o Hymenostylium recuroirostrum, en el extremo de varia- 


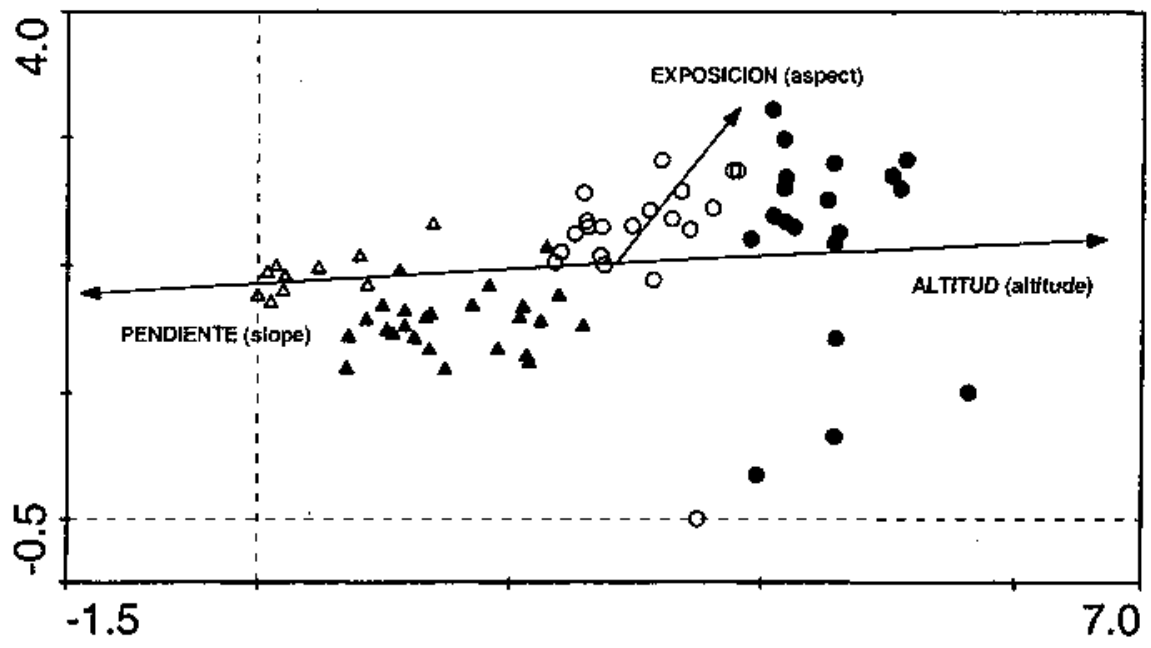

Figura 5. "Biplot" de ordenación del análisis DCA para 74 inventarios (las tendencias de las variables ambientales se incluyen como factores no ponderados). La correlación con el análisis TWINSPAN es: Triángulos: grupo A (A1: vacío; A2: relleno); Círculos: grupo B (B1: vacio; B2: relleno).

Figure 5. Ordination biplot of DCA analysis for 74 releves (Enoironmental variables tendencies are included as nonveighted factors). Correlation with TWISNPAN analysis is: Triangles: group A (A1: emply; A2; filled); Circles: group B (B1: empty; B2: filled).

ción más próximo a los inventarios de la clase Adiantetea (sector izquierdo del eje $\mathrm{x}$ ). En el extremo contrario, son Palustriella falcata y Philonotis calcarea las especies briofíticas que presentan un mayor peso o valor discriminante. Por otro lado, las especies comunes a un mayor número de inventarios se sitúan en la parte central del mismo eje. Tal es el caso de Palustriella commutata, Pinguicula grandifiora o Centaurium somedanum (Figura 6).

El análisis TWINSPAN clasificó los inventarios de referencia en los extremos de la matriz resultante. Los niveles de corte de pseudoespecies fueron 0,5 y 25. A partir de los valores de semejanza entre inventarios se establecieron grupos de inventarios para 1 y 2 niveles jerărquicos de clasificación, en función de las distancias entre ellos (medidas según el coeficiente phi). La interpretación del análisis pretende definir la variabilidad desde un punto de vista ecológico, mostrando las especies indicadoras que mejor se corresponden con los grupos seleccionados. Para ello se han creando dos tablas sintéticas con los valores de fidelidad de especies para cada grupo. Estas tablas fueron definidas según unos valores mínimos de fidelidad de 25 y 40 , para la división en 


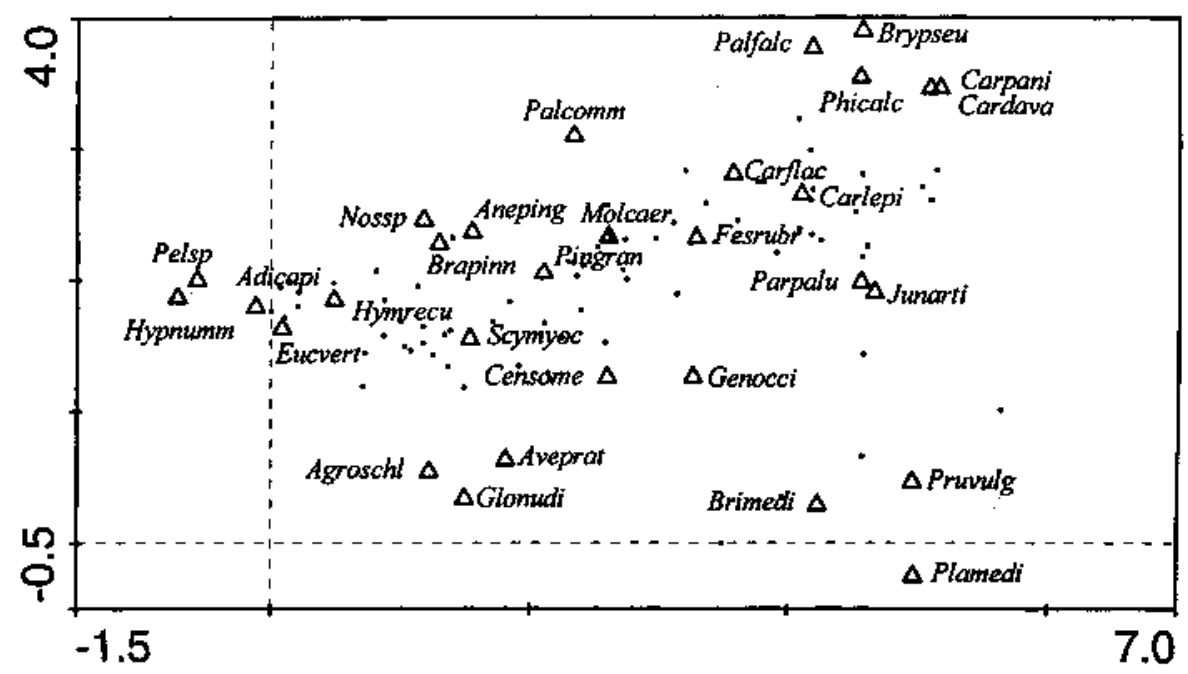

Figura 6. Biplot de ordenación de muestras (inventarios) y especies (No aparecen valores $<15)$.

Figure 6. Ordination biplot of samples (releves) and species (values < 15 don't appear).

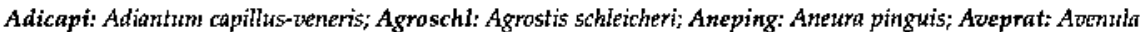
pratensis ssp. iberica var. tusconica; Brapinn: Brachypodtam pinnotum ssp. rupestre; Brimedi: Briza media: Brypseu; Bryum psewdorriquel num; Cardava: Carex daualliama; Carflac: Carex flacca; Ca rlepi: Corex lepidocarpa; Carpani: Carex panicen; Censome: Centaurium somedanum; Eucvert: Eucladiun verticillatum; Festubr: Festuca rubra; Genocit: Gentsta octidentalis; Glonudi: Globularia Mudidaulis; Hymecu; Hymenosiylinm recurutrostom;

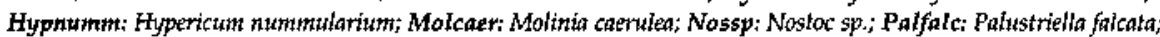
Junarti: Juncus artictlatus; Palcomm: Paiusirielia commulata; Parpaltu: Pamassia palusiris; Pelsp; Pellin sp:

Phicalc: Philonotis calcarea; Pingran: Pinguicula grandiflora; Plamedi: Plantago madit; Provulg: Prumella oulgaris; Scymyot: Scytontere myochrous.

2 y 4 grupos, respectivamente (Tabla 2). La división en cuatro grupos refleja la diversidad existente entre los inventarios.

Clasificación en dos grupos.- El grupo A incluye los 9 inventarios de referencia de la asociación Hyperico nummularii-Pinguiculetum coenocantabricae, además de 26 inventarios con C. somedanum, que corresponden a los de menor altitud, y señalan la transición hacia las paredes rezumantes colonizadas por dichas comunidades, todas ellas en pendientes próximas a $90^{\circ}$. Las especies más fieles son Adiantum capillus-veneris y Eucladium verticillatum, típicas de paredes rezumantes abrigadas de la clase Adiantetea Br.-Bl. 1942 (DÍAZ GONZÁLEZ al, 1982). El grupo B incluye 39 inventarios realizados en turberas planas y surgencias con formación de toba en situación de ladera. Casi todos los inventarios de este grupo proceden de cotas elevadas, presentando 
Tabla 2. Tablas sintéticas de los grupos definidos mediante el análisis TWINSPAN (para 2 y 4 grupos). Los valores corresponden al indice de fidelidad de las especies para cada grupo.

Tabte 2. Synoptic table of the cluster defined by TWINSPAN analysis (for 2 and 4 clusters). Values are species fidetity index for each different group.

\begin{tabular}{|c|c|c|}
\hline Grupo & $\mathbf{A}$ & $\mathbf{B}$ \\
\hline úmero de inventarios & 35 & 39 \\
\hline diantum capilus-veneris & 73.1 & - \\
\hline ucladium verticillatum & 68.7 & - \\
\hline ymenostylium fecurvirostrum & 59.8 & - \\
\hline grostis schleicheri & 43.5 & - \\
\hline Ilia sp. pl. & 36.8 & - \\
\hline ypericum nummilarium & 34.1 & - \\
\hline ampanula legionensis & 31.4 & - \\
\hline Hpatorium cannabinum & 31.4 & - \\
\hline venulla pratensis & 30.4 & - \\
\hline ngemannia atrowirens & 28.4 & - \\
\hline Erocoptis pyrenaica & 28.4 & - \\
\hline obularia nudicaulis & 27.2 & - \\
\hline quilegia vulgaris & 25.9 & - \\
\hline wharia hygrometrica & $25+2$ & - \\
\hline splenium trichomanes & 25.2 & - \\
\hline arex lepidocarpa & - & 67.7 \\
\hline armassia palustris & - & 67.7 \\
\hline nous articulatus & - & 55.7 \\
\hline iza media & - & 43.9 \\
\hline alustriella falcata & - & 43.7 \\
\hline unella vulgaris & - & 41.7 \\
\hline enista occidentalis & - & 41.4 \\
\hline antago media & - & 39.6 \\
\hline repis paitudosa & $=$ & 39.6 \\
\hline hilonotis calcarea & $=$ & 39.6 \\
\hline arex flacca & - & 38.1 \\
\hline altha palustris & $=$ & 37.4 \\
\hline arex datalliana & $=$ & 37.4 \\
\hline arex panicea & - & 37.4 \\
\hline yum pseudotriguetum & $=$ & 35.3 \\
\hline laginella selaginotides & $=$ & $35-3$ \\
\hline entha longifolia & - & 30.6 \\
\hline nagalis tenella & - & 30.6 \\
\hline dicularis mixta & - & 28.1 \\
\hline ystus compressus & - & 28.1 \\
\hline guisetumt uxiegatum & + & 28.1 \\
\hline hinanthus mitor & - & 28.1 \\
\hline olinia caerulea & - & 27.6 \\
\hline pipactis palustris & ᄀ & 25.5 \\
\hline ontodon spectes & + & 25.5 \\
\hline ifolium pratense & - & 25.5 \\
\hline
\end{tabular}

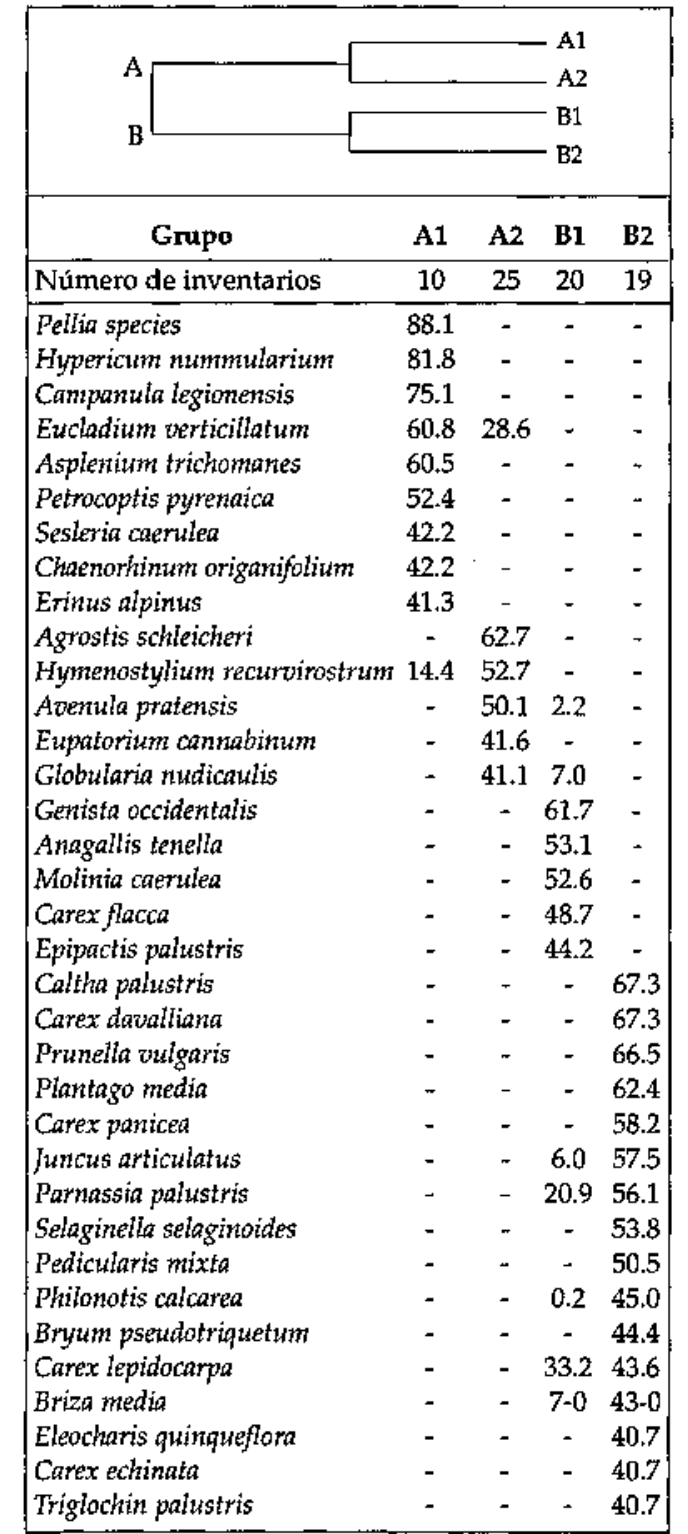

60 
comunidades relacionadas con turberas planas calcáreas de montaña (orden Caricetalia davallianae $\mathrm{Br}$ - $\mathrm{Bl}$. 1949), como las de los inventarios de referencia de la asociación Pinguiculo grandiflorae-Caricetum lepidocarpae.

Clasificación en cuatro grupos.- Separa cada uno de los grupos anteriores, A y B, en otros dos subgrupos. El grupo A1 está formado exclusivamente por los inventarios de la asociación Hyperico nummularii-Pinguiculetum coenocantabricae, los cuales presentan una importante variabilidad desde la zona de donde fue descrita, en los Picos de Europa, con plantas que no llegan a las montañas occidentales del área de estudio (como Hypericum nummularium o Campanula legionensis). El grupo A2 corresponde a comunidades similares, pero con especies cuya área de distribución es más amplia. En ambos casos se incluyen como especies acompañantes plantas típicas de las paredes y extraplomos calizos limítrofes, como Petrocoptis pyrenaica ssp. giaucifolia o Globularia nudicaulis. Los otros dos grupos, B1 y B2, difieren entre sí principalmente en la pendiente; mientras que el grupo B1 incluye los inventarios realizados en surgencias de pendiente donde es frecuente la presencia de toba, el grupo B2 se corresponde estrictamente con turberas planas, situadas en bajas pendientes y a mayor altitud.

La correspondencia entre los inventarios y las poblaciones a las que pertenecen se muestran en la Tabla 3 , asi como los valores de frecuencia y fidelidad que definen las especies diagnósticas y frecuentes de cada grupo. Como es lógico, algunas de las plantas que no figuran como diagnósticas para ningún grupo son comunes a la mayor parte de inventarios y marcan la relación. ecológica existente entre las comunidades estudiadas; las más frecuentes son Pinguicula grandiflora (presente en el $78 \%$ del total de inventarios), Centaurium somedanum (76\%) y Carex lepidocarpa (78\%).

\section{Discusión}

Implicaciones del hábitat en la conseroación de $\mathrm{C}$. somedanum

El carácter estenoico de $\mathrm{C}$. somedanum puede ser una valiosa herramienta para su conservación. Comparando las comunidades vegetales en que vive con su autoecología, es posible definir su valencia ecológica y por tanto, caracterizar su hábitat potencial. Se ha determinado así la estrecha relación de C. somedanum con surgencias y otros ambientes relacionados, donde existen unos requerimientos ecológicos estrictos de humedad y $\mathrm{pH}$, concretamente fuentes donde se liberan aguas muy carbonatadas. Estas fuentes, colonizadas en su origen por comunidades briofiticas dominadas por Palustriella commu- 
PIRINEOS 160

Tabla 3. Número de inventarios de cada población asignados a los grupos definidos por TWINSPAN, y principales especies diagnósticas (valores 0-100) y constantes (en \%) definidas para cada grupo. N. Inv: número de inventarios; S1/S2: grupo asignado para $2 / 4$ divisiones.

Table 3. Number of releves assigned to the main clusters defined by TWINSPAN divisions, and principal diagnostic (values $0-100$ ) and constant (\%) species defined for each group. $N$. Inv.: number of releves; $S 1 / S 2 ;$ assigned group for $2 / 4$ cluster divisions.

\begin{tabular}{|c|c|c|c|c|c|c|}
\hline Pob. & Nombre & Altitud & Pendiente & N. Inv. & S1 & $S 2$ \\
\hline I & La Malva (subpoblación 1) & 610 & $90^{\circ}$ & 1 & $\mathbf{A}$ & A1 \\
\hline $\mathbf{I}$ & La Malva (subpoblación 1 y 2) & $595 / 740$ & $80 / 90^{\circ}$ & 19 & $\mathbf{A}$ & A2 \\
\hline 1 & La Malva (subpoblación 3) & $740 / 750$ & $25^{\circ}$ & 9 & $\mathbf{B}$ & B1 \\
\hline 3 & Pto. de Somiedo & 1400 & $10^{\circ}$ & 3 & B & B2 \\
\hline 4 & Valle del lago & 1300 & $10^{\circ}$ & 2 & $\mathbf{B}$ & $\mathrm{B} 2$ \\
\hline 4 & Valle del lago & 1300 & $10^{\circ}$ & 5 & B & B1 \\
\hline 5 & Torre de Babia 1 & 1500 & $40^{\circ}$ & 3 & B & B1 \\
\hline 6 & Torre de Babia 2 & 1700 & $10^{\circ}$ & 2 & $\mathbf{B}$ & B2 \\
\hline 9 & Valle Arroxos & 1350 & $40^{\circ}$ & 2 & B & B1 \\
\hline 10 & Murias Lon & 1600 & $10^{\circ}$ & 5 & B & B2 \\
\hline 12 & Aquiru & 920 & $80 / 90^{\circ}$ & 3 & $\mathbf{A}$ & $\mathrm{A} 2$ \\
\hline 13 & Catu & 1000 & $40 / 50^{\circ}$ & 3 & $\mathbf{A}$ & A2 \\
\hline 14 & Som & 1300 & $10^{\circ}$ & 1 & B & B2 \\
\hline \multicolumn{7}{|c|}{ Especies diagnósticas } \\
\hline \multicolumn{7}{|c|}{$\begin{array}{l}\text { S1- Grupo A: Adiantum capillus-veneris (73); Eucladium verticilatum (68); } \\
\text { Hymenostylium recurvirostrum (59). Grupo B: Parnassia palustris (67), Carex lepidocarpa } \\
\text { (67), Juncus articulatus (55). } \\
\text { \$2-Grupo A1: Pellia sp. pl. (88), Hypericum nummularium (81), Campanula legionensis } \\
\text { (75). Grupo A2: Agrostis schleicheri (62), Hymenostylium recurvirostrum (52), Avenula } \\
\text { pratenstis subsp. iberica var. vasconica (50). Grupo B1: Genista occidentalis (61) Anagalis } \\
\text { tenella (53), Molinia caerulea subsp. caerulea (52). Grupo B2: Carex davalliana (67), } \\
\text { Caltha palustris (67). Prunella vulgaris (66), Plantago media (62). }\end{array}$} \\
\hline \multicolumn{7}{|c|}{ Especies constantes } \\
\hline \multicolumn{7}{|c|}{$\begin{array}{l}\text { S1 - Grupo A: Pinguicula grandiflora (74), Centaurium somedanum (71), Adiantum capi- } \\
\text { llus-veneris (69), Eucladium verticillatum (63). Grupo B: Carex lepidocarpa (90), } \\
\text { Pinguicula grandifora (82), Centaurium somedanum (79), Parnassia palustris (64). } \\
\text { S2 - Grupo A1: Eucladium verticillatum (100), Pinguicula grandiflora (90), Adiantum } \\
\text { capillus-veneris (90). Grupo A2; Centaurium somedanum (96), Pinguicula grandiflora } \\
\text { (68), Agrostis schleicheri (64), Avenula pratensis subsp. iberica var. vasconica (60). Grupo } \\
\text { B1: Pinguicula grandiflora (100), Centaurium somedanum (100), Genista occidentalis (95), } \\
\text { Carex lepidocarpa (85). Grupo B2: Carex lepidocarpa (95), Parnassia palustris (79), Juncus } \\
\text { articulatus (68), Pinguicula grandiflora (63). }\end{array}$} \\
\hline
\end{tabular}


tata, permiten el desarrollo de las comunidades fontinales, y por tanto de las plantas especialmente adaptadas a estos medios. La caracterización de las diferentes variables que determinan la existencia de surgencias (contactos litológicos, etc.) junto a las variables ya descritas para algunas de las especies que en ellas viven (altitud, pendiente, etc.) puede ser utilizada en la selección futura de áreas potencialmente adecuadas para C. somedanum y las comunidades en que vive.

La demografía de las poblaciones presenta también una estrecha relación con el hábitat, provocando una distribución contagiosa - variabilidad espacial- y cambios en la disponibilidad de hábitat interanual -variabilidad temporal -. Estas circunstancias se derivan de unas surgencias con caudal muy variable, en función del régimen de precipitaciones, lo cual dificulta la realización de censos. Todo ello incide sobre la tasa de mortalidad de las poblaciones, las cuales funcionan como metapoblaciones, con ciclos variables de extinción local y recolonización (HANSKI, 1999) que conviene tener en cuenta ante futuros estudios demográficos.

\section{Riesgo y amenazas}

Por todo lo dicho, la escasez y especificidad del hábitat son los principales factores responsables de la propia rareza de C. somedanum, por lo que las limitaciones y amenazas que se ciernen sobre dicho hábitat condicionan las posibilidades de expansión de la planta. En todo caso, la dispersión, reducida extensión y bajo número de individuos de las poblaciones pueden ser tomados como factores de riesgo biológico para la viabilidad de la especie. Además, la reducción e incluso desaparición de algunas poblaciones que hemos podido observar se debió a la construcción de carreteras, captaciones a través de tubos de extracción y otras causas que afectan a la disponibilidad de agua. Asimismo, algunas de las poblaciones de ambientes higroturbosos están sometidas a una intensa presiôn ganadera, lo que provoca la alteración fisico-química del ecosistema en que vive la planta, y su consiguiente degradación. Se trata de las principales amenazas reales en la actualidad, por cuanto nunca hemos observado ni predación ni ramoneo en las poblaciones de nuestra gencianácea endémica.

\section{Conclusiones}

Se ha observado una declinación del $35 \%$ en el número de localidades donde se había citado Centaurium somedanum, en un tiempo máximo de 15 
años. Ha desaparecido de Villanueva (Teverga, Asturias); Sograndio (Proaza, Asturias) y San Martín de Ondes (Belmonte, Asturias), con el agravante de que en algunas de estas localidades la eliminación completa del hábitat ha provocado su extinción; no obstante, no podemos descartar la existencia de otras poblaciones en los alrededores. También se observó durante el periodo de estudio una seria reducción del hábitat en el Puerto de Somiedo (Cabrillanes, León) y en el Valle del Lago (Somiedo, Asturias), debido a la reciente construcción de infraestructuras y extracción de agua.

El hábitat potencial de $\mathrm{C}$. someḋanum se define como "ambientes próximos a surgencias de aguas carbonatadas, especialmente en zonas marginales del agua circulante, donde se acumula el precipitado de carbonato cálcico". La rareza, dispersión espacial y escasa superficie de estos biotopos limitan la disponibilidad de hábitat de esta especie y, por tanto, su posible expansión. Teniendo en cuenta que por ahora no ha sido encontrada en otro tipo de hábitat (salvo individuos aislados que circundan su hábitat principal, y no legan a constituir poblaciones estables), se confirma como una planta fontinal especializada, con una reducida valencia ecológica.

C. somedanum es además una planta muy estenoica, indicadora de medios ricos en altas concentraciones de precipitados de carbonato cálcico. Siempre se integra en complejos de vegetación fontinal originados por manantiales de aguas carbonatadas; precisamente estos últimos figuran como hábitat prioritario en la directiva europea $97 / 62 / \mathrm{CEE}$, señalado con el código 7220 , y con los códigos $\mathrm{C} 2, \mathrm{C} 2.1, \mathrm{C} 2.12$ y C2.12.1 en la codificación EUNIS (2003). Otros hábitats que bordean surgencias en terrenos calcáreos se corresponden con el código 7230 (D4, D4.1, D4.13, D4.1F y D4.1N en la codificación EUNIS). Pero en la Cordillera Cantábrica unos y otros resultan escasos y vulnerables.

En función de los datos recogidos y según los criterios de la UICN (2001), C. somedanum entra en la categoría de vulrerable (VU), tanto a nivel regional como mundial, según los criterios $\mathrm{A} 2 c ; \mathrm{C} 1 ; \mathrm{y} 2$; ello implica la existencia de un riesgo claro de extinción en estado silvestre. De hecho, los criterios referentes a la declinación y área geográfica de las poblaciones (A2; B) sitúan el grado de amenaza de C. somedanum muy próximo a la siguiente categoría de amenazada, "en peligro" (EN).

Como es natural, el mantenimiento de $C$. somedanum está ligado a la conservación de las comunidades en que vive, las cuales albergan también otras especies raras o amenazadas de la Cordillera Cantábrica, como Triglochin palustris, Equisetum variegatum, Cochlearia pyrenaica, Juncus cantabricus, Potentilla fruticosa o Swertia perennis. La presencia de estas plantas representa un valor añadido que justifica el estudio y conservación de dichas comunidades. 
Los estudios futuros sobre C. somedanum deben tener en cuenta no solo aspectos ecológicos de los biotopos en que vive, sino también demográficos, relacionados con las metapoblaciones. Recomendamos el estudio y seguimiento de sus poblaciones, así como las oportunas medidas de conservación:

-protección del hábitat, es decir, mantenimiento de los sistemas que alimentan surgencias calcáreas, control de las extracciones de agua, etc.

-protección de las poblaciones conocidas, considerándolas de acceso restringido en los espacios protegidos en que viven y cartografiándolas al detalle para poder controlar las actividades ganaderas, evitar infraestructuras perjudiciales, etc.

\section{Agradecimientos}

Agradecemos la colaboración de Carmen Fernández Ordóñez en la determinación de briófitos, la de Victor Vázquez ent la localización de nuevas citas de C. somedantum y la de Susana Fernández en la interpretación geológica de surgencias. Al Parque Natural de Somiedo y al Gobierno del Principado de Asturias reconocemos su apoyo para la realizaciốn de los trabajos de campo, y a la Consejería de Medio Ambiente de la Junta de Castilla y León su interés en la conservación de esta gentianácea. También agradecemos a dos evaluadores anónimos sus oportunas sugerencias.

\section{Referencias}

AEDO, C. (1990). Informe sobre la flora amenazada de Asturias. Agencia del Medio Ambiente del Principado de Asturias. 338 pp. (inédito).

BAN̄ARES BAUDET, A. (coord). (2002). Biología de la conservación de plantas amenazadas. Organismo Autónomo Parques Nacionales. Ministerio de Medio Ambiente. Madrid.

BANARES, A., BLANCA, G., GÜEMES, J., MORENO, J. C., ORTIZ, S. (2003). Atlas y Libro Rojo de la Flora Vascular Amenazada de España. Direcciôn General de Conservación de la Naturaleza. Madrid.

BRAUN-BLANQUET, J. (1979). Fitosociología. Bases para el estudio de las comunidades vegetales. $\mathrm{H}$. Blume Ed. Madrid.

CARLÓN, M., MAYOR, M., LASTRA, J.J. (2003). Atlas corológico de la flora asturiana, I. Bol. Cien. Nat. R.I.D.E.A, 48: 77-110. 
DÍAZ GONZÁLEZ, T. E., GUERRA, J. \& NIETO, J. M. (1982). Contribución al conocimiento de la clase Adiantetea Br-Bl. 1942 en la Península Ibérica. Anales Jard. Bot. Madrid, 38(2): 497-506.

DÍAZ GONZÁLEZ, T. E., FERNÁNDEZ PRIETO, J. A. (1994). La vegetación de Asturias. Itinera Geobotanica, 8: 243-528.

DÍAZ GONZÁLEZ, T. E., FERNÁNDEZ PRIETO, J. A., NAVA FERNÁNDEZ, H.S., BUENO SANCHEZ, A. (2003). Flora en peligro. Asturnatura, 19.

DDXON, K. R. AND CHAPMAN, J. A. (1980). Harmonic mean measure of animal activity areas. Ecology, 61(5): 1040-1044.

EUNIS Database v2, 2003 (version 2005). European Nature Information System. European Environment Agency. http:/eunis.finsiel,ro/eunis /index.jsp

FERNÁNDEZ CASAS, R. GAMARRA \& M. J. MORALES ABAD (eds.). (1994). Asientos corológicos de la flora occidental, 21. Fontqueria, 39: 281394.

FERNÁNDEZ PRIETO, J. A. (1978). Notas sobre la flora somedana. Notas florísticas y ecológicas sobre la flora ibérica. II. Universidad de Oviedo.

HANSKI, I. A. (1999). Metapopulation ecology. Oxford University Press. New York.

HILL, M. O. (1979). TWINSPAN, a fortran program for detrended correspondence analysis and reciprocal averaging. Ecology and Systematics. Cornell University, Ithaca, NY, USA.

KEITH, D. A. (1998). An evaluation and modification of World Conservation Union Red List criteria for classification of extinction risk in vascular plants. Conservation Biology, 12: 1076-1090.

LAINZ, M. (1976). Aportaciones al conocimiento de la flora Cántabro-Astur, XI (1). Bol. Cien. Nat. R.I.D.E.A, 22: 32.

LASTRA, J. J. (1989). Estudio de la flora y vegetación vascular del concejo de Grado y sus contornos. Tesis doctoral inédita. Unversidad de Oviedo. $405 \mathrm{pp}$.

LEPS, J. \& SMILAUER, P. (2003). Multivariate Analysis of Ecological Data using CANOCO. Cambridge University Press.

MANSION, G. \& STRUWE, L. (2004). Generic delimitation and phylogenetic relationships within the subtribe Chironiinae (Chironieae: Gentianaceae), with special reference to Centautium: evidence from nrDNA and cpDNA sequences. Molecular Phylogenetics and Evolution, 32: 951-977.

MANSION, G. (2004). A new classification of the polyphyletic genus Centaurium Hill (Chironiinae, Gentianaceae): description of the New World endemic Zeltnera, and reinstatement of Gyrandra Griseb. and Schenkia Griseb. Taxon, 53 (3): 719-740.

MARRERO, M. V., BAÑARES, A., CARQUE, E. (2003). Plant resource conservation planning in protected natural areas: an example from the Canary Islands, Spain. Biological Conservation, 113: 339-410. 
MENGES, E. S. \& GORDON, D. R. (1996). Three levels of monitoring intensity for rare plants species. Natural Areas Joumal, 16: 227-237.

PUENTE, E. (1988). Flora y vegetación de la cuenca alta del río Sil. Instituto Fray Bernardino de Sahagún. Diputación Provincial de León.

RIVAS-MARTÍNEZ, S., DÍAZ GONZÁLEZ, T. E., FERNÁNDEZ PRIETO, J. A., LOIDI, J., PENAS, Á. (1984). La vegetación de la alta montaña cantábrica. Los Picos de Europa. Ed. Leonesas. León.

RIVAS-MARTÍNEZ, S. DÍAZ GONZÁLEZ, T. E., FERNÁNDEZ GONZÁLEZ, F., LOIDI, J., LOUSA, M. \& PENAS, A. (2002). Vascular plant communities of Spain and Portugal. Itinera Geobotanica, 15 (I): 5-432.

ROMERO RODRÍGUEZ, C. M. (1977). Datos para la flora de la cuenca alta del río Luna. Bol. Est. Centr. de Ecologia, 6 (11): 25-39.

ROMERO RODRIGUEZ, C. M. (1984). Flora y vegetación de la ctienca alta del rio Luna. Monografias ICONA, 29. Madrid. 273 pp.

TICHY, L. (2002). JUICE, software for vegetation classification. Joumal of Vegetation Science, 13: 451-453.

UICN (2001). Categorias y Criterios de la Lista Roja de la UICN: Versión 3.1. Comisión de Supervivencia de Especies de la UICN. UICN, Gland (Suiza) y Cambridge (Reino Unido). ii +33 pp.

VALADON, A. (2003). Le plan d'action flore vasculaire du Parc National des Pyrénées: stratégie, méthodes et premiers resultats. Acta Botanica Barcinonensia, 49: 127-146.

VV. AA. (2000). Lista roja de flora vascular española. (valoración según categorías UICN). Conservación vegetal, 6 (extra).

ZELTNER, L. (1970). Recherches de biosystématique sur les genres Blackstonia Huds. et Centaurium Hill (Gentianacées). Bulletin de la Société Neuchateloise des Sciences Naturelles, 93: 1-164. 